\title{
FILTROS NO LINEALES PARA RECONSTRUIR SEÑALES DE ELECTROCARDIOGRAMAS
}

\section{NONLINEAR FILTERS TO RECONSTRUCT ELECTROCARDIOGRAM SIGNALS}

\author{
SABA INFAnTE* LUIS SÁNCHEZ ${ }^{\dagger}$ FERnANDo CEDEÑO ${ }^{\ddagger}$
}

Received: 7/May/2012; Revised: 19/May/2014;

Accepted: 21/May/2014

*Departamento de Matemáticas, Centro de Análisis, Modelado y Tratamiento de Datos (CAMYTD), Facultad de Ciencia y Tecnología, Universidad de Carabobo. Valencia, Venezuela. E-Mail: sinfante@uc.edu.ve

${ }^{\dagger}$ Departamento de Matemáticas, Facultad de Ciencias de la Educación, Universidad de Carabobo. Valencia, Venezuela. E-Mail: Isanchez8@uc.edu.ve

${ }^{\ddagger}$ Departamento de Matemáticas, Centro de Análisis, Modelado y Tratamiento de Datos (CAMYTD), Facultad de Ciencia y Tecnología, Universidad de Carabobo. Valencia, Venezuela. E-Mail: fjcedeno@uc.edu.ve 


\begin{abstract}
Resumen
Las señales de los electrocardiogramas han sido usadas en patologías cardíacas para detectar enfermedades del corazón. El objetivo principal de este trabajo es proponer técnicas de filtraje de señales para reducir el ruido, extraer información, reconstruir los estados, y propiedades morfológicas de los latidos del corazón. Adicionalmente se pretende representar la actividad cardíaca en forma simple, informativa, precisa, y de fácil interpretación para los Cardiólogos. Para lograr estos objetivos se proponen implementar los siguientes algoritmos: filtro de partículas genérico (FPG), filtro de partículas con remuestreo (FPR), filtro de Kalman sin esencia (FKSE), y el filtro de partículas sin esencia (FPSE), considerando la estructura básica del modelo dinámico sintético de McSharry et al. (2003) [16]. Los resultados demuestran que los filtros se desempeñan muy bien en la reconstrucción de los estados del sistema del ritmo cardíaco, aun introduciendo pequeñas variaciones en las varianzas de los ruidos de la ecuación de observación; es decir, los métodos tiene la capacidad de reproducir la señal original del modelo sintético simulado y del modelo sintético con datos reales en forma precisa. Finalmente se evalúa el desempeño de los filtros en términos de la desviación estándar empírica, observándose poca variabilidad entre los errores estimados y una rápida ejecución de los algoritmos.
\end{abstract}

Palabras clave: modelo sintético ECG; filtros no lineales; morfología de las ondas.

\begin{abstract}
ECG signals have been used in cardiac pathology to detect disease heart. The main objective of this paper is to propose signal filtering techniques to reduce noise, extract information, to reconstruct the states and properties Morphological heartbeat. In addition, aims to represent the cardiac activity in a simple, informative, accurate, and easy to interpret for cardiologists. To achieve these objectives are proposed to implement the following algorithms: generic particle filter (GPF), resampling particle filter (RPF), unscented Kalman filter (UKF) and the unscented particle filter (UFP) considering the basic structure of synthetic dynamic model McSharry et al. (2003) [16]. The results show that filter performs very well in the reconstruction of the states heart rate system, while introducing small variations in the variances of the noises of the equation observation, ie, the methods have the ability to reproduce the original signal the synthetic model simulated and the synthetic model with real data accurately. Finally evaluates the performance of the filters in terms of the empirical standard deviation, showing little variability among the estimated errors and fast execution of algorithms.
\end{abstract}


Keywords: synthetic ECG model; nonlinear filters; morphology of waves.

Mathematics Subject Classification: 62L12.

\section{Introdución}

El corazón esta formado por un músculo que tiene la capacidad de generar impulsos eléctricos a través de un sistema de conducción, que se propaga a través de las aurículas y ventrículos, con el propósito de contraerse en forma rítmica. Las variaciones del potencial eléctrico durante el ciclo cardíaco producen las ondas características del electrocardiograma (ECG); su análisis es de gran importancia porque describe la cronología de los eventos cardíacos. Formalmente un ECG es un registro gráfico que describe la actividad eléctrica producida por el flujo de la corriente iónica que causan la contracción de las fibras del corazón y que posteriormente permite un estado de relajación. El ECG se obtiene mediante el registro de la diferencia de potencial entre varios electrodos colocados en la superficie corporal, este proceso se registra en papel milimetrado en forma de ondas, siendo las principales, las ondas identificadas por: $P, Q, R, S$ y $T$, las cuales se repiten periódicamente en una persona sana, con las frecuencias de los latidos cardíacos. En la Figura (1) se muestra un latido simulado utilizando el modelo McSharry et al. (2003) [16], donde se evidencian las ondas antes mencionadas.

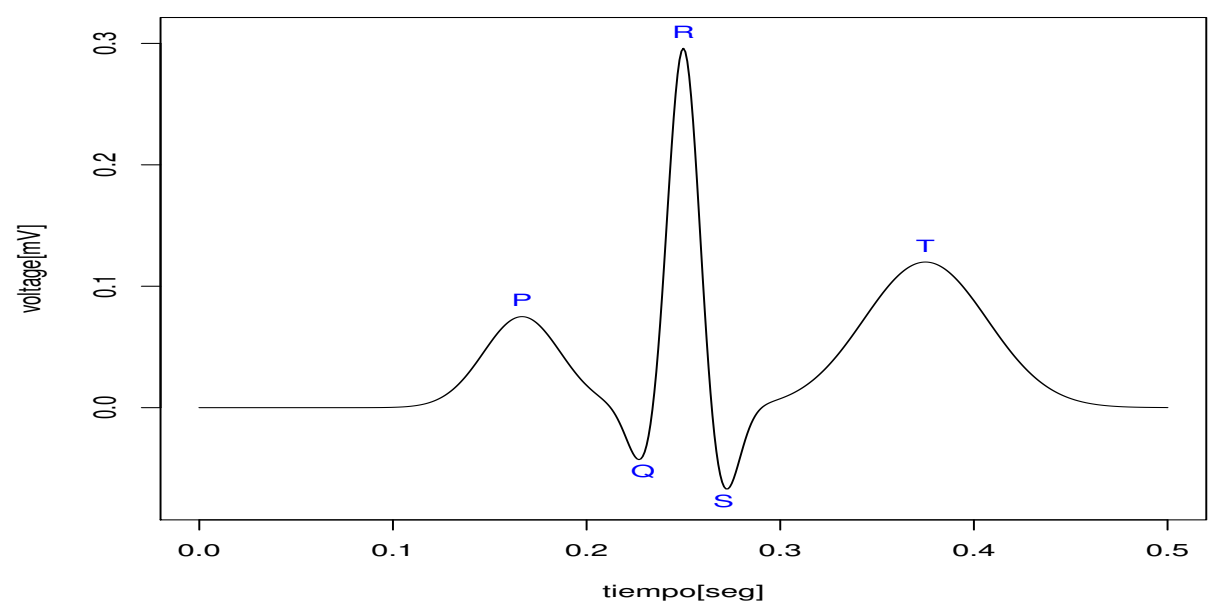

Figura 1: Señal típica del ECG. 
La onda $P$ es una deflexión positiva por encima de la línea base provocada por la depolarización auricular manifestada en el corazón por la contracción auricular y es muy útil en el estudio de las arritmias, su duración normal es aproximadamente de 0.10 segundos. El complejo $Q R S$ comprende 3 ondas: $Q, R, S$, y representa la depolarización del miocardio ventricular. La onda $Q$ representa el potencial de acción que viaja a través del septum interventricular, las ondas $R$ y $S$ indican contracción del miocardio, las anormalidades en el complejo $Q R S$ pueden indicar: taquicardia de origen ventricular, hipertrofia ventricular u otras anormalidades ventriculares, su duración normal es aproximadamente igual a 0.06 segundos a 0.10 segundos. La onda $T$ representa la repolarización de los ventrículos.

El procesamiento de señales se realiza con el propósito de mejorar la precisión de las mediciones, reproducir la señal cuando se compara con las mediciones manuales, y cuando la extracción de la información no se obtiene en forma clara. Por otra parte, los registros del ECG pueden ser contaminados por muchas fuentes de error, tales como: interferencias eléctricas en los equipos, contacto incorrecto de los electrodos, músculo contraído, movimiento en los equipos cuando se realiza el ECG, errores en el proceso de conversión digital, etc. Dado que estas interferencias morfológicamente se confunden con la actividad cardíaca, se requiere emplear técnicas de filtrado que permitan la extracción exacta y efectiva de la información. Los métodos de filtrado preservan las características clínicas importantes mientras que proporcionan una alta reducción del ruido (Sayadi et al. (2010) [27]). Las técnicas utilizadas hasta ahora para filtrar y eliminar el ruido son: descomposición de ondículas (Nikolaev et al. (2000) [18]), análisis por componentes principales (Paul et al. (2000) [19], Moody \& Mark (1989) [17]), análisis de componentes independientes (Potter et al. (2002) [20], Barros et al. (1998) [3], He et al. (2006)) [10], reducción de ruido no lineal (Schreiber \& Kaplan (1996) [28]), redes neuronales (Clifford \& Tarassenko (2001) [6]). Recientemente se han utilizado las técnicas del filtro de Kalman extendido, (Sayadi y Sameni (2007) [23], Sayadi y Shamosollahi (2008a) [24], Sayadi y Shamosollahi (2008b) [25], Sayadi y Shamosollahi (2009) [26], Sameni et al. (2005) [21], filtro de Kalman extendido suavizado (Sayadi et al. (2010) [27]) y filtro de Kalman sin esencia (Vepa (2010) [33]), entre otros. Dado que las señales generadas por los latidos del corazón tienen un comportamiento no lineal, el objetivo principal de este trabajo es reconstruir el modelo dinámico sintético electrocardiograma (ECG) desarrollado por McSharry et al. (2003) [16], usando las técnicas de filtros de partículas (FP) y alguna de sus modificaciones. Hay muchas variantes de estos métodos, en este trabajo se implementarán los siguientes algoritmos: filtro de partículas genérico 
(FPG), filtro de partículas con remuestreo (FPR), el filtro de Kalman sin esencia (FKSE) y el filtro de partículas sin esencia (FPSE), sobre el sistema dinámico discretizado propuesto por McSharry et al. (2003) [16]. Para una revisión extensa de estos métodos se recomienda revisar los trabajos de: Arulampalam et al. (2002) [2], Gordon et al. (1993) [9], Doucet et al. (2000) [7], Storvik (2002) [30], Doucet et al. (2001) [8], Simon (2006) [29], Andrieu et al. (2010) [1], van der Merwe et al. (2000) [31], van der Merwe (2004) [32], Vepa (2010) [33], y Sánchez \& Infante (2013) [22], entre otros.

El resto del artículo es organizado como sigue. En la Sección 2 se resumen: los materiales y métodos; en la Sección 3 se muestran los resultados obtenidos; y en la Sección 4 se realiza una discusión de los resultados y se establecen unas conclusiones.

\section{Materiales y métodos}

Un sistema dinámico es un proceso que consiste en un conjunto de posibles estados, bajo un conjunto de reglas que determinan los estados presentes en términos de los estados pasados. La relación entre las observaciones $\mathbf{y}_{t} \mathbf{y}$ las variables de estados desconocidos $\mathbf{x}_{t}$ se puede representar en forma compacta por un sistema dinámico estocástico no lineal en tiempo discreto, como sigue:

$$
\begin{aligned}
& \mathbf{x}_{t}=\mathcal{M}_{t}\left(\mathbf{x}_{t-1}\right)+\mathbf{u}_{t} ; \quad \mathbf{u}_{t} \sim \mathcal{N}\left(0, Q_{t}\right) \\
& \mathbf{y}_{t}=\mathcal{H}_{t}\left(\mathbf{x}_{t}\right)+\mathbf{v}_{t} ; \quad \mathbf{v}_{t} \sim \mathcal{N}\left(0, R_{t}\right)
\end{aligned}
$$

La ecuación dada en (1), se conoce como la ecuación de estado, $\mathcal{M}_{t}$ es un operador de transición que mapea el espacio de estados dentro del mismo espacio de estados, $\mathbf{x}_{t} \in \mathcal{X} \subset \mathbb{R}^{n}$ denota un vector de estados desconocidos en un tiempo $t, \mathbf{u}_{t}$ es un vector de errores aleatorios de estimación de los estados, y $Q_{t}$ es la matriz de covarianza de los errores de los estados. Por otra parte, la ecuación dada en (2), representa la ecuación de observación, $\mathbf{y}_{t} \in \mathcal{Y} \subset \mathbb{R}^{n}$ denota un vector de observaciones, $\mathcal{H}_{t}$ es un operador que mapea el espacio de los estados dentro del espacio de las observaciones, $\mathbf{v}_{t}$ es un vector de errores aleatorios cometido en las mediciones de las observaciones, y $R_{t}$ es la matriz de covarianza de los errores de las observaciones. Una tarea importante es estimar los estados desconocidos $\mathbf{x}_{0: t}=\left(x_{0}, \ldots, x_{t}\right)$, basándose en las medidas obtenidas a partir del proceso de observación $\mathbf{y}_{1: t}=\left(y_{1}, \ldots, y_{t}\right)$. La idea principal es estimar recursivamente en el tiempo $t$ la distribución a posteriori $p\left(\mathbf{x}_{0: t} \mid \mathbf{y}_{1: t}\right)$, y sus características asociadas incluyendo la distribución marginal $p\left(x_{t} \mid \mathbf{y}_{1: t}\right)$, la esperanza a posteriori $E\left(x_{t} \mid \mathbf{y}_{1: t}\right)$, y la varianza a posteriori $\operatorname{Var}\left(x_{t} \mid \mathbf{y}_{1: t}\right)$, entre 
otras cantidades de interés. En algún tiempo $t$, se puede estimar la distribución a posteriori usando el Teorema de Bayes:

$$
p\left(\mathbf{x}_{0: t} \mid \mathbf{y}_{1: t}\right) \propto \frac{p\left(\mathbf{y}_{1: t} \mid \mathbf{x}_{0: t}\right) p\left(\mathbf{x}_{0: t}\right)}{\int p\left(\mathbf{y}_{1: t} \mid \mathbf{x}_{0: t}\right) p\left(\mathbf{x}_{0: t}\right) d \mathbf{x}_{0: t}}
$$

En la práctica interesa estimar la distribución marginal $P\left(x_{t} \mid \mathbf{y}_{1: t}\right)$, que satisface la siguiente forma recursión:

Paso 1 Predicción:

$$
p\left(x_{t} \mid \mathbf{y}_{1: t-1}\right)=\int p\left(x_{t} \mid x_{t-1}\right) p\left(x_{t-1} \mid \mathbf{y}_{1: t-1}\right) d x_{t-1}
$$

Paso 2 Actualización:

$$
p\left(x_{t} \mid \mathbf{y}_{1: t}\right)=\frac{p\left(y_{t} \mid x_{t}\right) p\left(\mathbf{x}_{t} \mid \mathbf{y}_{1: t-1}\right)}{p\left(y_{t} \mid \mathbf{y}_{1: t-1}\right)}
$$

donde:

$$
p\left(y_{t} \mid \mathbf{y}_{1: t-1}\right)=\int p\left(y_{t} \mid x_{t}\right) p\left(x_{t} \mid \mathbf{y}_{1: t-1}\right) d x_{t}
$$

En general hay problemas para calcular las ecuaciones (4), y (5). En este trabajo se proponen las siguientes técnicas de filtrado de señales para estimar la distribución a posteriori marginal de los estados: filtro de partículas genérico, filtro de partículas con remuestreo, filtro de Kalman sin esencia, y filtro de partículas sin esencia.

\subsection{Filtro de partículas genérico}

Supóngase que se simulan $N$ muestras independientes idénticamente distribuidas llamadas partículas de una variable aleatoria, que se denota por: $\left\{\mathbf{x}_{0: t}^{(i)}, i=1, \ldots, N\right\}$ de acuerdo a una distribución $p\left(\mathbf{x}_{0: t} \mid \mathbf{y}_{1: t}\right)$. Un estimador empírico para está distribución es dado por:

$$
\tilde{p}_{N}\left(\mathbf{x}_{0: t} \mid y_{1: t}\right)=\frac{1}{N} \sum_{i=1}^{N} \delta\left(\mathbf{x}_{0: t}-\mathbf{x}_{0: t}^{(i)}\right)
$$

donde $\delta\left(\mathbf{x}_{0: t}-\mathbf{x}_{0: t}^{(i)}\right)$ denota un punto de masa de la función Delta de Dirac localizada en $\mathbf{x}_{0: t}^{(i)}$. En esto consiste el método de muestreo de importancia (MI). El MI 
puede ser modificado para obtener el estimador $\tilde{p}_{N}\left(\mathbf{x}_{0: t} \mid \mathbf{y}_{1: t}\right)$ de $p\left(\mathbf{x}_{0: t} \mid \mathbf{y}_{1: t}\right) \sin$ modificar las trayectorias simuladas $\left\{\mathbf{x}_{0: t}^{(i)}, i=1, \ldots, N\right\}$. Esto significa considerar una función de importancia $q\left(\mathbf{x}_{0: t} \mid \mathbf{y}_{1: t}\right)$ en el tiempo $t$ que admite una distribución marginal en el tiempo $t-1$, digamos:

$$
q\left(\mathbf{x}_{0: t} \mid \mathbf{y}_{1: t}\right)=q\left(\mathbf{x}_{0: t-1} \mid \mathbf{y}_{1: t-1}\right) q\left(x_{t} \mid \mathbf{x}_{0: t-1}, \mathbf{y}_{1: t}\right)
$$

iterando, se obtiene:

$$
q\left(\mathbf{x}_{0: t} \mid \mathbf{y}_{1: t}\right)=q\left(x_{0}\right) \prod_{k=1}^{t} q\left(x_{k} \mid \mathbf{x}_{0: k-1}, \mathbf{y}_{1: k}\right)
$$

Entonces la función de importancia permite evaluar recursivamente en el tiempo los pesos de importancia:

$$
w_{t}^{(i)} \propto w_{t-1}^{(i)} \frac{p\left(y_{t} \mid x_{t}^{(i)}\right) p\left(x_{t}^{(i)} \mid x_{t-1}^{(i)}\right)}{q\left(x_{t}^{(i)} \mid \mathbf{x}_{0: t-1}^{(i)}, \mathbf{y}_{1: t}\right)}
$$

Un caso particular, es considerar que la distribución a priori $p\left(\mathbf{x}_{0: t}\right)$ como una distribución de importancia, esto es:

$$
q\left(\mathbf{x}_{0: t} \mid \mathbf{y}_{1: t}\right)=p\left(\mathbf{x}_{0: t}\right)=p\left(x_{0}\right) \prod_{k=1}^{t} p\left(x_{k} \mid x_{k-1}\right)
$$

En este caso el peso de importancia satisface:

$$
w_{t}^{(i)} \propto w_{t-1}^{(i)} p\left(y_{t} \mid x_{t}^{(i)}\right)
$$

El algoritmo del filtro de partículas genérico (FPG) se resume:

Paso 1. Inicialización: para $t=0 ;$ y $i=1, \ldots, N$; se muestrea:

- $x_{0}^{(i)} \sim p\left(x_{0}\right)$

- $w_{0}^{(i)} \sim p\left(y_{0} \mid x_{0}^{(i)}\right)$

- $\tilde{w}_{0}^{(i)}=\frac{w_{0}^{(i)}}{\sum_{j=1}^{N} w_{0}^{(j)}}$.

Paso 2. Muestreo de importancia: para $t=1,2, \ldots, T$; y $i=1, \ldots, N$ : 
- $x_{t}^{(i)} \sim q\left(x_{t} \mid \mathbf{x}_{0: t-1}^{(i)}, \mathbf{y}_{1: t}\right)$ y se forma el conjunto:

$$
\mathbf{x}_{0: t}^{(i)}=\left(\mathbf{x}_{0: t-1}^{(i)}, x_{t}^{(i)}\right)
$$

- Se evalúan los pesos de importancia:

$$
w_{t}^{(i)} \propto w_{t-1}^{(i)} \frac{p\left(y_{t} \mid x_{t}^{(i)}\right) p\left(x_{t}^{(i)} \mid x_{t-1}^{(i)}\right)}{q\left(x_{t}^{(i)} \mid \mathbf{x}_{0: t-1}^{(i)}, \mathbf{y}_{1: t}\right)}
$$

- Se normalizan los pesos de importancia:

$$
\tilde{w}_{t}^{(i)}=\frac{w_{t}^{(i)}}{\sum_{j=1}^{N} w_{t}^{(j)}}
$$

Paso 3. Salidas: distribución a posteriori, valor esperado y la covarianza a posteriori.

$$
\begin{gathered}
\tilde{p}_{N}\left(\mathbf{x}_{0: t} \mid y_{1: t}\right) \approx \sum_{i=1}^{N} \tilde{w}_{0: t}^{(i)} \delta\left(\mathbf{x}_{0: t}-\mathbf{x}_{0: t}^{(i)}\right) \\
\tilde{x}_{t}=E\left(x_{t} \mid y_{1: t}\right) \approx \sum_{i=1}^{N} \tilde{w}_{t}^{(i)} x_{t}^{(i)} \\
\tilde{P}_{t} \approx \sum_{i=1}^{N} \tilde{w}_{t}^{(i)}\left(x_{t}^{(i)}-\tilde{x}_{t}\right)\left(x_{t}^{(i)}-\tilde{x}_{t}\right)^{T}
\end{gathered}
$$

\subsection{Filtro de partículas con remuestreo}

El problema que se presenta con el algoritmo de filtro de partículas genérico es el fenómeno de la degeneración. Es conocido que después de unas pocas iteraciones las partículas pueden tener pesos despreciables lo que implica, que la varianza de los pesos de importancia pueden incrementarse en el tiempo. Doucet et al. (2000) [7], establecieron dos proposiciones que permiten seleccionar una función de importancia que minimiza la varianza de los pesos sobre las trayectorias de los estados simulados $\left\{\mathbf{x}_{0: t}^{(i)}\right\}$. Una medida adecuada para solventar el problema de degeneración de los pesos es calcular el tamaño de muestra efectiva 
( $\left.N_{T M E}\right)$ introducido en Kong et al. (1994) [14] y Liu (1996) [15], y que se define como:

$$
N_{T M E}=\frac{N}{1+\operatorname{Var}_{q\left(\cdot \mid \mathbf{y}_{1: t}\right)}\left(\hat{w}_{t}^{*(i)}\right)}
$$

en la práctica es complicado calcular $N_{T M E}$, pero se estima por:

$$
\hat{N}_{T M E}=\frac{1}{\sum_{i=1}^{N}\left[\tilde{w}_{t}^{(i)}\right]^{2}}
$$

entonces se compara $\hat{N}_{T M E}$ con un umbral prefijado $M_{U}=\frac{N}{2}$ (Chen (2003) [4]). El algoritmo modificado se resume como sigue:

Paso 1. Inicialización: para $t=0$ y $i=1, \ldots, N$; se muestrea:

- $x_{0}^{(i)} \sim p\left(x_{0}\right)$

- $w_{0}^{(i)} \sim p\left(y_{0} \mid x_{0}^{(i)}\right)$

- $\tilde{w}_{0}^{(i)}=\frac{w_{0}^{(i)}}{\sum_{j=1}^{N} w_{0}^{(j)}}$.

Paso 2. Muestreo de importancia: para $t=1, \ldots, T$; y $i=1, \ldots, N$ :

- $x_{t}^{(i)} \sim q\left(x_{t} \mid \mathbf{x}_{0: t-1}^{(i)}, \mathbf{y}_{1: t}\right)$; y se construye el conjunto:

$$
\mathbf{x}_{0: t}^{(i)}=\left\{\mathbf{x}_{0: t-1}^{(i)}, x_{t}^{(i)}\right\}
$$

- Se evalúan los pesos de importancia:

$$
w_{t}^{(i)} \propto w_{t-1}^{(i)} \frac{p\left(y_{t} \mid x_{t}^{(i)}\right) p\left(x_{t}^{(i)} \mid x_{t-1}^{(i)}\right)}{q\left(x_{t}^{(i)} \mid \mathbf{x}_{0: t-1}^{(i)}, \mathbf{y}_{1: t}\right)}
$$

- Se normalizan los pesos de importancia:

$$
\tilde{w}_{t}^{(i)}=\frac{w_{t}^{(i)}}{\sum_{j=1}^{N} w_{t}^{(j)}}
$$

- Se evalúa:

$$
\hat{N}_{T M E}=\frac{1}{\sum_{i=1}^{N}\left[\tilde{w}_{t}^{(i)}\right]^{2}}
$$


Paso 3. Remuestreo:

- Si $\hat{N}_{T M E} \geq M_{U}$, se hace $\mathbf{x}_{0: t}^{(i)}=\tilde{x}_{0: t}^{(i)}$, para $i=1, \ldots, N$.

- Si $\hat{N}_{T M E}<M_{U}$, para $i=1, \ldots, N$ se muestrea un índice $j(i)$ distribuido de acuerdo a la distribución discreta con $N$ elementos que satisfacen $p_{r}\{j(i)=l\}=\tilde{w}_{t}^{(l)}$, para $l=1, \ldots, N$. Entonces se hace $\mathbf{x}_{0: t}^{(i)}=\tilde{x}_{0: t}^{j(i)}$ y $\tilde{w}_{t}^{(i)}=\frac{1}{N}$.

Paso 4. Las salidas del algoritmo se obtienen usando las ecuaciones dadas en (15), (16) y (17).

\subsection{Transformación sin esencia}

La transformación sin esencia (TSE) es un método para calcular los estadísticos de primer y segundo orden como: la media y la covarianza de una variable aleatoria que sufre de una transformación no lineal $y=f(x)$ y se basa en el principio probabilístico que dice que es más fácil aproximar una distribución de probabilidad que una función no lineal arbitraria (Julier y Uhlmann (2004)); es una forma elegante y exacta de calcular la media y la covarianza de la función $y$ mediante la expansión de una serie de Taylor. Sea $\mathbf{x}$ un vector de dimensión $n_{x}$ que tiene media $\bar{x}$ y matriz de varianza covarianza $P_{x x}$. La TSE calcula la media y la covarianza de $y=f(x)$ como sigue:

Paso 1 Determinísticamente se generan $2 n_{x}+1$ puntos sigmas $S_{i}=\left\{x_{i}, w_{i}\right\}$ :

- Se calcula $x_{0}=\bar{x}$, para $i=0$.

- Se generan $x_{i}$ :

$$
\begin{aligned}
& x_{i}=\bar{x}+\left(\sqrt{\left(n_{x}+\lambda\right) P_{x x}}\right)_{i} ; \quad i=1, \ldots, n_{x} \\
& x_{i}=\bar{x}-\left(\sqrt{\left(n_{x}+\lambda\right) P_{x x}}\right)_{i} ; i=n_{x}+1, \ldots, 2 n_{x}
\end{aligned}
$$

- Se calculan $w_{i}^{(m)}$ y $w_{i}^{(c)}$ de la siguiente manera:

$$
\begin{aligned}
w_{i}^{(m)} & =\frac{\lambda}{n_{x}+\lambda} \quad ; \quad i=0 \\
w_{i}^{(c)} & =w_{0}^{(m)}+\left(1-\alpha^{2}+\beta\right) \quad ; \quad i=0 \\
w_{i}^{(c)} & =w_{i}^{(m)}=\frac{1}{\left[2\left(n_{x}+\lambda\right)\right]} \quad ; \quad i=1, \ldots, 2 n_{x}
\end{aligned}
$$

donde:

$$
\lambda=\alpha^{2}\left(n_{x}+\kappa\right)-n_{x}
$$


Paso 2 Se propagan los puntos sigmas a través de la TSE:

$$
y_{i}=f\left(x_{i}\right) \quad ; \quad i=0, \ldots, 2 n_{x} .
$$

Paso 3 Se calcula la media y la covarianza de $y=f(x)$ :

$$
\bar{y}=\sum_{i=1}^{2 n_{x}} w_{i}^{(m)} y_{i} \quad ; \quad P_{y y}=\sum_{i=1}^{2 n_{x}} w_{i}^{(c)}\left(y_{i}-\bar{y}\right)\left(y_{i}-\bar{y}\right)^{T} .
$$

$\lambda$ es un parámetro de escala que determina la dirección de los puntos sigma; $\alpha$ mide la dispersión de los puntos sigma alrededor de la media $\bar{x}(0 \leq \alpha \leq 1) ; \kappa$ es un segundo parámetro de escala que varía entre $0<\kappa<3-n_{x} ; \beta$ representa los grados de libertad extra usado para introducir algún conocimiento a priori de la distribución de $x_{i}$, además es el tercer parámetro que incorpora los efectos de orden superior de la distribución; $\left(\sqrt{\left(n_{x}+\lambda\right) P_{x x}}\right)_{i}$ es la $i$-ésima fila o columna de la raíz cuadrada de la matriz $\left(n_{x}+\lambda\right) P_{x x}$ y $w_{i}$ son un pesos asociados con el $i$-ésimo punto sigma tal que $\sum_{i=0}^{2 n_{x}} w_{i}=1$. La exactitud de la media y la covarianza de $y=f(x)$ es garantizada mediante la expansión de la serie de Taylor, independientemente de la forma de $f(x)$. Los puntos sigma generados garantizan la convergencia debido al mecanismo del método Monte Carlo, dado en Julier (2000) [11].

\subsection{Filtro de Kalman sin esencia}

El FKSE es un estimador de error cuadrático medio mínimo que se usa como alternativa al filtro de Kalman extendido (FKE) para estimar las señales que tienen un comportamiento no lineal. El FKSE debe su nombre de la transformación sin esencia desarrollada por Julier \& Uhlmann (2004) [13]. En el FKSE la distribución de los estados es representada por una variable aleatoria Gaussiana que se especifica mediante un conjunto mínimo de puntos muestrales escogidos determinísticamente. Se aumenta el modelo espacio estado hasta que incluya las componentes de los estados originales y las variables ruidos $x_{t}^{a}=\left(x_{t}^{T} u_{t}^{T}\right)^{T}$. El esquema de selección de los puntos sigma es aplicado a este nuevo vector de estados aumentados para calcular la correspondiente matriz sigma $x_{i, t}^{a}$. Luego el filtro actualiza la media y la covarianza mediante una aproximación usando las ecuaciones del filtro de Kalman (ver a van der Merwe et al. (2000) [31]), para mayores detalles. El FKSE se resume, para un sistema dinámico no lineal discreto general:

$$
\begin{aligned}
& \mathbf{x}_{\mathbf{t}}=f\left(\mathbf{x}_{t-1}\right)+u_{t} \\
& \mathbf{y}_{\mathbf{t}}=h\left(\mathbf{x}_{t}\right)+v_{t}
\end{aligned}
$$


donde: $u_{t} \sim N\left(0, Q_{t}\right)$, y $v_{t} \sim N\left(0, R_{t}\right) . \mathbf{x}_{\mathbf{t}} \in \mathbb{R}^{n_{x}}$ es un vector de estados $n_{x} \times 1, y_{t} \in \mathbb{R}^{m}$ es un vector de medidas $m \times 1, u_{t} \in \mathbb{R}^{q}$ es un vector de ruidos de los estados $q \times 1$, y $v_{t} \in \mathbb{R}^{r}$ es un vector de ruidos de las observaciones $r \times 1$; como sigue:

Paso 1. Inicialización: para $t=0$.

$$
\begin{aligned}
\hat{x}_{t}^{a} & =E\left(x_{t}^{a}\right) \\
P_{t}^{a} & =E\left[\left(x_{t}^{a}-\hat{x}_{t}^{a}\right)\left(x_{t}^{a}-\hat{x}_{t}^{a}\right)^{T}\right] .
\end{aligned}
$$

Paso 2. Generación de los puntos sigma: para $t=1, \ldots, T$

$$
x_{i, t}^{a}=\left(\left[\hat{x}_{t}^{a} \quad ; \quad \hat{x}_{t}^{a}+\sqrt{\left(n_{x}+q+\lambda\right) P_{t}^{a}} \quad ; \quad \hat{x}_{t}^{a}-\sqrt{\left(n_{x}+q+\lambda\right) P_{t}^{a}}\right]\right)_{i} .
$$

Paso 3. Propagación:

$$
\begin{aligned}
x_{i, t+1}^{a} & =f\left(x_{i, t}^{a}\right) \\
\hat{x}_{t+1}^{-} & =\sum_{i=0}^{2\left(n_{x}+q\right)} w_{i}^{(c)} x_{i, t+1}^{a} \\
P_{t+1}^{-} & =\sum_{i=0}^{2\left(n_{x}+q\right)} w_{i}^{(m)}\left(x_{i, t+1}^{a}-\hat{x}_{t+1}^{-}\right)\left(x_{i, t+1}^{a}-\hat{x}_{t+1}^{-}\right)^{T} \\
y_{i, t+1} & =h\left(x_{i, t+1}^{a}\right) \\
\hat{y}_{t+1}^{-} & =\sum_{i=0}^{2\left(n_{x}+q\right)} w_{i}^{(c)} y_{i, t+1} \\
P_{t+1}^{y y} & =\sum_{i=0}^{2\left(n_{x}+q\right)} w_{i}^{(m)}\left(y_{i, t+1}-\hat{y}_{t+1}^{-}\right)\left(y_{i, t+1}-\hat{y}_{t+1}^{-}\right)^{T}+R_{t} \\
P_{t+1}^{x y} & =\sum_{i=0}^{2\left(n_{x}+q\right)} w_{i}^{(m)}\left(x_{i, t+1}^{a}-\hat{x}_{t+1}^{-}\right)\left(y_{i, t+1}-\hat{y}_{t+1}^{-}\right)^{T} .
\end{aligned}
$$

Paso 4. Actualización:

$$
\begin{aligned}
K_{t+1} & =P_{t+1}^{x y}\left(P_{t+1}^{y y}\right)^{-1} \\
\hat{x}_{t+1}^{+} & =\hat{x}_{t+1}^{-}+K_{t+1}\left(y_{t}-\hat{y}_{t}^{-}\right) \\
P_{t+1}^{+} & =P_{t+1}^{-}-K_{t+1} P_{t+1}^{y y} K_{t+1}^{T} .
\end{aligned}
$$




\subsection{Filtro de partículas Kalman sin esencia}

El nuevo filtro que resulta de usar el FKSE dentro de la estructura de los filtros de partículas (FP) es llamado filtro de partícula sin esencia (FPSE). La idea principal es usar el FKSE para generar las muestras de la distribución propuesta requerida en el FP, es decir:

$$
x_{t}^{(i)} \sim q\left(x_{t}^{(i)} \mid \mathbf{x}_{0: t-1}, \mathbf{y}_{1: t}\right)^{(i)}=N\left(\hat{x}_{t}^{(i)}, \hat{P}_{t}^{(i)}\right)
$$

donde $\hat{x}_{t}^{(i)}$ y $\hat{P}_{t}^{(i)}$ es la media y la covarianza de los puntos sigma $x_{i}$, generados por el procedimiento del FKSE. Un ciclo del FPSE puede ser resumido como sigue:

Paso 1. Inicialización: en un tiempo $t=0, \mathrm{y} i=1, \ldots, N$.

- $x_{0}^{(i)} \sim p\left(x_{0}\right)$

- $w_{0}^{(i)}=p\left(y_{0} \mid x_{0}^{(i)}\right)$

- $\tilde{w}_{0}^{(i)}=\frac{w_{0}^{(i)}}{\sum_{j=1}^{N} w_{0}^{(j)}}$.

- $\hat{x}_{0}^{(i)}=E\left(x_{0}^{(i)}\right)$

- $P_{0}^{(i)}=E\left\{\left(x_{0}^{(i)}-\hat{x}_{0}^{(i)}\right)\left(x_{0}^{(i)}-\hat{x}_{0}^{(i)}\right)^{T}\right\}$

- $x_{0}^{a,(i)}=\left(\begin{array}{c}x_{0}^{(i)} \\ w_{0}\end{array}\right)$

- $P_{0}^{a,(i)}=\left(\begin{array}{cc}P_{0}^{(i)} & 0 \\ 0 & Q_{0}^{(i)}\end{array}\right)$.

Paso 2. Muestreo de Importancia: para $t=1, \ldots, T$ y $i=1, \ldots, N$;

- Se actualizan las partículas con el FKSE, para estimar la media y la covarianza; las cuales se aproximan generando los puntos sigma.

- Se calculan los puntos sigmas:

$$
\begin{aligned}
& x_{t}^{a,(i)}=\left[\hat{x}_{t}^{a,(i)} ; \quad \hat{x}_{t}^{a,(i)}+\sqrt{\left(n_{x}+q+\lambda\right) P_{t}^{a,(i)}} ;\right. \\
& \left.\hat{x}_{t}^{a,(i)}-\sqrt{\left(n_{x}+q+\lambda\right) P_{t}^{a,(i)}}\right] \text {. }
\end{aligned}
$$


- Predicción:

$$
\begin{aligned}
x_{j, t+1}^{x,(i)} & =f\left(x_{t}^{a,(i)}\right) \\
\hat{x}_{t+1 \mid t}^{(i)} & =\sum_{j=0}^{2\left(n_{x}+q\right)} w_{j}^{(m)} x_{j, t+1}^{x,(i)} \\
P_{t+1 \mid t}^{(i)} & =\sum_{j=0}^{2\left(n_{x}+q\right)} w_{j}^{(c)}\left(x_{j, t+1}^{x,(i)}-\hat{x}_{t+1 \mid t}^{(i)}\right)\left(x_{j, t+1}^{x,(i)}-\hat{x}_{t+1 \mid t}^{(i)}\right)^{T} \\
y_{j, t+1}^{(i)} & =h\left(x_{j, t+1}^{x,(i)}\right) \\
\hat{y}_{t+1 \mid t}^{(i)} & =\sum_{j=0}^{2\left(n_{x}+q\right)} w_{j}^{(m)} y_{j, t+1}^{(i)} \\
P_{t+1}^{v v} & =\sum_{j=0}^{2\left(n_{x}+q\right)} w_{j}^{(c)}\left(y_{j, t+1}^{(i)}-\hat{y}_{t+1 \mid t}^{(i)}\right)\left(y_{j, t+1}^{(i)}-\hat{y}_{t+1 \mid t}^{(i)}\right)^{T}+R_{t} \\
P_{t+1}^{x x} & =\sum_{j=0}^{2\left(n_{x}+q\right)} w_{j}^{(c)}\left[x_{j, t+1}^{x,(i)}-\hat{x}_{t+1 \mid t}\right]\left[y_{j, t+1}^{(i)}-\hat{y}_{t+1 \mid t}^{(i)}\right]^{T} \\
K_{t+1} & =P_{t+1}^{x y}\left(P_{t+1}^{v v}\right)^{-1} \\
\hat{x}_{t+1}^{(i)} & =\hat{x}_{t+1 \mid t}^{(i)}+K_{t+1}\left[y_{t}-h\left(\hat{x}_{t+1 \mid t}^{(i)}\right)\right] \\
\hat{P}_{t+1}^{(i)}= & P_{t+1 \mid t}^{(i)}-K_{t+1} P_{t+1}^{v v} K_{t+1}^{T} .
\end{aligned}
$$

- Para $i=1, \ldots, N$; se muestrea de la densidad de importancia:

$$
\tilde{x}_{t}^{(i)} \sim N\left(\hat{x}_{t}^{(i)}, \hat{P}_{t}^{(i)}\right)
$$

y se forma el conjunto $\tilde{x}_{0: t}^{(i)}=\left(x_{0: t-1}^{(i)}, \tilde{x}_{t}^{(i)}\right)$.

- Para $i=1, \ldots, N$; se evalúan los pesos:

$$
w_{t}^{(i)}=p\left(y_{t} \mid x_{t}^{(i)}\right) \hat{w}_{t-1}^{(i)} .
$$

- Para $i=1, \ldots, N$; se normalizan los pesos de importancia:

$$
\hat{w}_{t}^{(i)}=\frac{w_{t}^{(i)}}{\sum_{j=1}^{N} w_{t}^{(j)}} .
$$

- Se evalúa $\hat{N}_{T M E}$. 
Paso 3. Remuestreo: si $\hat{N}_{T M E}<M_{U}$ se remuestrea, a partir de la población $\left\{\tilde{x}_{0: t}^{(i)}, \hat{w}_{t}^{(i)}\right\}$, y se obtiene un nuevo conjunto $\left\{\tilde{x}_{0: t}^{(i)}, \frac{1}{N}\right\}$ con pesos uniformes.

Paso 4. Las salidas del algoritmo se obtienen usando las ecuaciones dadas en (15), (16) y (17).

Para validar los resultados obtenidos se usará como medida de adecuación, la desviación estándar empírica (DEE), definida por:

$$
D E E(x)=\sqrt{\operatorname{Var}\left(x_{t \mid l}\right)}=\frac{1}{N} \sum_{t=1}^{N}\left(\frac{1}{M} \sum_{j=1}^{M}\left(x_{t \mid l}^{(j)}-x_{t}^{(j)}\right)^{2}\right)^{\frac{1}{2}}
$$

donde: $x_{t}^{(j)}$ es el estado verdadero para la $j$-ésima simulación; $x_{t \mid l}^{(j)}=\sum_{i=1}^{N} \tilde{w}_{t \mid l}^{(i)} x_{t}^{j,(i)}$, es el estimador Monte Carlo de $x_{t \mid t}=E\left(x_{t} \mid y_{1: t}\right)$ para la $j$-ésima señal de prueba; $x_{t}^{j,(i)}$ es la $i$-ésima trayectoria simulada asociada con la señal $j$; y $\tilde{w}_{t \mid t}^{(i)}=\hat{w}_{t}^{(i)}$ es el peso de importancia. El propósito de utilizar el criterio de la desviación estándar empírica es proveer respuestas confiables y eficientes de los resultados y determinar que tan apropiada es la aproximación implementada a la solución esperada.

\subsection{El modelo dinámico sintético}

McSharry et al. (2003) propusieron un modelo sintético del ECG usando un conjunto de ecuaciones de estados que genera una trayectoria tridimensional con coordenadas $(x, y, z)$. El modelo consiste en un ciclo límite circular de radio uno en el plano $(x, y)$, el cual impulsa la trayectoria hacia arriba y hacia abajo mientras se aproxima al punto de inflexión en el ECG. Las formas de las ondas son descrita por procesos Gaussianos que corresponden atractores positivos y negativos en la dirección del eje $z$. La casi-periócidad del modelo es reflejada por los movimientos de la trayectoria alrededor del ciclo límite, mientras la variación interlatidos del corazón es reproducida usando los movimientos de la trayectoria en la dirección del eje $z$. El modelo es definido por un conjunto de tres ecuaciones diferenciales ordinarias: 


$$
\begin{aligned}
& \dot{x}=\alpha x-\omega \\
& \dot{y}=\alpha y+\omega x \\
& \dot{z}=-\sum_{i \in\{P, Q, R, S, T\}} a_{i} \Delta \theta_{i} \exp \left(-\frac{\Delta \theta_{i}^{2}}{2 b_{i}^{2}}\right)-\left(z-z_{0}\right)
\end{aligned}
$$

donde: $\dot{x}=\frac{d x}{d t}, \dot{y}=\frac{d y}{d t}, \dot{z}=\frac{d z}{d t}, \alpha=1-\sqrt{x^{2}+y^{2}}, \Delta \theta_{i}=\left(\theta-\theta_{i}\right) \bmod 2 \pi$, $\theta=\operatorname{atan} 2(y, x), \theta \in[-\pi, \pi], \omega=2 \pi f$ es la velocidad angular de la trayectoria, y $f$ es la frecuencia entre latidos del ritmo cardíaco. Cada uno de los picos $P, Q, R, S, T$ son modelados usando una distribución Gaussiana localizada en la posición angular de $\theta=\left(\theta_{P}, \theta_{Q}, \theta_{R}, \theta_{S}, \theta_{T}\right)$. Los parámetros $a_{i}, b_{i}$, y $\theta_{i} ; i \in\{P, Q, R, S, T\}$, corresponden a la amplitud, anchura (tiempo de duración), y centro de los parámetros. La línea base del ECG es modelada usando el parámetro $z_{0}(t)=A \sin \left(2 \pi f_{2} t\right)$, que se supone que es un componente de baja amplitud sinusoidal acoplado con la frecuencia respiratoria $f_{2}$ (McSharry et al. (2003) [16]), donde $A=0.15 \mathrm{mV}$. La coordenada $z$ de esta trayectoria es representada gráficamente contra el tiempo para obtener el ECG sintético. Sameni et al. (2005) [21] transformó las ecuaciones dinámicas dadas en (36), en la forma polar de la siguiente manera:

$$
\begin{aligned}
\dot{r} & =r(1-r) \\
\dot{\theta} & =\omega \\
\dot{z} & =-\sum_{i \in\{P, Q, R, S, T\}} a_{i} \Delta \theta_{i} \exp \left(-\frac{\Delta \theta_{i}^{2}}{2 b_{i}^{2}}\right)-\left(z-z_{0}\right)
\end{aligned}
$$

donde: $\dot{r}=\frac{d r}{d t}, \dot{\theta}=\frac{d \theta}{d t}, \mathrm{y} \dot{z}=\frac{d z}{d t}$. El nuevo conjunto de ecuaciones tiene algunos beneficios comparado con las ecuaciones originales. La forma polar es muy simple de interpretar, la primera ecuación puede ser omitida ya que no afecta al resto de las ecuaciones, y los parámetros de fase esta dada en forma explícita lo que facilita la localización angular de las ondas $P, Q, R, S$ y $T$. El modelo dado en la ecuación (37) en la forma espacio estado discreta es:

$$
\begin{aligned}
& \theta_{t+1}=\theta_{t}+\omega \delta \\
& z_{t+1}=z_{t}-\sum_{i \in\{P, Q, R, S, T\}} \delta a_{i} \Delta \theta_{i} \exp \left(-\frac{\Delta \theta_{i}^{2}}{2 b_{i}^{2}}\right)+\eta
\end{aligned}
$$

donde: $\delta$ es un tiempo de muestreo, $\theta$ es una señal que tiene forma de diente de sierra que se espera que sea cero en los $R$ picos, y es de forma lineal entre $-\pi$ y $\pi$ para las muestras de ECG entre dos $R$ picos sucesivos, y $\eta$ es un ruido aleatorio que representa los efectos del paseo de riesgo base y algunas otras fuentes 
de error relacionadas con el modelo. Los parámetros $a_{i}, b_{i}$ y $\theta_{i}$ son la amplitud, dispersión angular y localizaciones de las funciones Gaussianas usadas para modelar cada componente del ECG. Para usar los filtros propuestos se debe colocar los modelos en la forma espacio estado, se consideran $\theta$ y $z$ como variables de estados y los parámetros más importantes del modelo son:

$$
w_{t}=\left\{a_{P}, \ldots, a_{T}, b_{P}, \ldots, b_{T}, \theta_{P}, \ldots, \theta_{T}, \omega, \eta\right\}
$$

donde: $w_{t}$ es un vector de ruido del proceso, con matriz de covarianza $Q_{t}=$ $E\left(w_{t} w_{t}^{T}\right)$. La relación entre la ecuación de estado y la ecuación de observación depende de la localización de los electrodos $\phi_{t}$ en el paciente y los errores cometidos en las mediciones $m_{t}$ y se relacionan con el vector de estados, por:

$$
\left(\begin{array}{c}
\phi_{t} \\
m_{t}
\end{array}\right)=\left(\begin{array}{ll}
1 & 0 \\
0 & 1
\end{array}\right)\left(\begin{array}{c}
\theta_{t} \\
z_{t}
\end{array}\right)+\left(\begin{array}{c}
u_{t}^{(1)} \\
u_{t}^{(2)}
\end{array}\right)
$$

donde:

$$
u_{t}=\left(\begin{array}{c}
u_{t}^{(1)} \\
u_{t}^{(2)}
\end{array}\right)
$$

es un vector de ruidos de la ecuación de observación, con matriz de covarianza $R_{t}=E\left(u_{t} u_{t}^{T}\right)$. Las matrices $Q_{t}$ y $R_{t}$ se consideran independientes y diagonales. Finalmente, para relacionar los parámetros del modelo con los registros del ECG real, se definen los $a_{i}$ como:

$$
a_{i}=\frac{\alpha_{i} \omega}{b_{i}^{2}} \quad ; \quad i \in\{P, Q, R, S, T\} .
$$

Por otra parte, dado que se usa una mezcla de cinco Gaussianas para estimar las ondas $P, Q, R, S, \mathrm{y} T$, se define una variable aleatoria con distribución normal como sigue: si $x \sim N\left(\mu, \sigma^{2}\right)$ entonces la función de densidad de probabilidad viene dada por:

$$
g\left(x ; \mu ; \sigma^{2}\right)=\frac{1}{\sqrt{2 \pi \sigma^{2}}} \exp \left(-\frac{(x-\mu)^{2}}{2 \sigma^{2}}\right)
$$

donde: $\frac{1}{\sqrt{2 \pi \sigma^{2}}}, \mu$ y $\sigma^{2}$ determinan la amplitud, el centro y la dispersión de la función $g$ respectivamente. Dado que los picos de las ondas del ECG corresponden a los centros de funciones Gaussianas se define $\mu=\theta_{i}$, para determinar las localizaciones de los picos como sigue:

$$
P=\theta_{P} \quad ; \quad Q=\theta_{Q} \quad ; \quad R=\theta_{R} \quad ; \quad S=\theta_{S} \quad ; \quad T=\theta_{T} .
$$


Análogamente, $b_{i}=\sigma$ especifica la desviación estándar de la posición de los picos de $\theta_{i}$. La desviación define los puntos iniciales y finales de la mezcla de densidades que describe el proceso multimodal PQRST, este resultado se mantiene siempre y cuando se suponga simetría para cada onda del ECG.

\section{Resultados}

En este trabajo se implementaron en lenguaje de programación Matlab los siguientes algoritmos: FPG, FPR, FKSE, y FPSE, para la reconstrucción de una señal generada por el modelo sintético de un ECG propuesto por McSharry et al. (2003) [16], y un modelo real de ECG de señales obtenidas de un individuo sano tomado de la base de datos physionet que se encuentra disponible en la siguiente dirección: http://www.physionet.org/physiobank/database/nsrdb/. Por otra parte, el modelo sintético considerado fue discretizado por el método de Euler de primer orden, considerando $x_{t+1}=x_{t}+h f\left(x_{t}\right)$, con un paso de tamaño $h=0.003$. La ecuación de estado discretizada resultante es:

$$
\begin{aligned}
& x_{t+1}=x_{t}+h\left(\alpha x_{t}-\omega y_{t}\right)+u_{1 t} \\
& y_{t+1}=y_{t}+h\left(\alpha y_{t}+\omega x_{t}\right)+v_{1 t} \\
& z_{t+1}=z_{t}+h\left(-\sum_{i \in\{P, Q, R, S, T\}} a_{i} \Delta \theta_{i} \exp \left(-\frac{\Delta \theta_{i}^{2}}{2 b_{i}^{2}}\right)-\left(z_{t}-z_{0}\right)\right)+e_{t}
\end{aligned}
$$

donde: $u_{1 t} \sim N\left(0, \sigma_{u 1}^{2}\right), v_{1 t} \sim N\left(0, \sigma_{v 1}^{2}\right)$, y $e_{t} \sim N\left(0, \sigma_{e}^{2}\right)$. Por lo general no se disponen de las observaciones experimentales del sistema dinámico que se quiere estudiar, entonces las observaciones requeridas para evolucionar se generan a través de una ecuación de observación lineal (ecuación de observación) sugerida por Chui y Chen (2009) [5]; es decir:

$$
\mathbf{r}_{t+1}=\mathbf{x}_{t}+\zeta_{t}
$$

donde: $\mathbf{r}_{t}=\left(x_{t}^{\prime}, y_{t}^{\prime}, z_{t}^{\prime}\right)^{T}, \mathbf{x}_{t}=\left(x_{t}, y_{t}, z_{t}\right)^{T}, \mathrm{y} \zeta_{t} \sim N\left(\mathbf{0}, \sigma_{\zeta}^{2} \mathbf{I}\right), \mathbf{0}$ es un vector de ceros, y donde la matriz I es la matriz identidad. Para inicializar el FPG, consideramos las siguientes distribuciones a priori: $x_{0}=1, y_{0}=0, z_{0}=0.04$, $u_{1 t} \sim N\left(0,10^{-3}\right), v_{1 t} \sim N\left(0,10^{-3}\right), e_{t} \sim N\left(0,10^{-3}\right), \zeta_{t} \sim N(\mathbf{0}, 10 \mathbf{I})$. Para inicializar el FPR, consideramos las siguientes distribuciones a priori: $x_{0}=1$, $y_{0}=0, z_{0}=0.04, u_{1 t} \sim N\left(0,10^{-3}\right), v_{1 t} \sim N\left(0,10^{-3}\right), e_{t} \sim N\left(0,10^{-4}\right)$, $\zeta_{t} \sim N(\mathbf{0}, 10 \mathbf{I})$. En los FPG y FPR se eligió como función de importancia una 
distribución normal, como se elige en Arulampalam et al. (2002). Los valores iniciales para FKSE fueron los siguientes:

$$
\begin{gathered}
\hat{x}_{0}^{+}=(1,0,0.04) \quad, \quad P_{0}^{+}=\left(\begin{array}{lll}
1 & 0 & 0 \\
0 & 1 & 0 \\
0 & 0 & 1
\end{array}\right) \\
Q_{t}=\left(\begin{array}{ccc}
0.0001 & 0 & 0 \\
0 & 0.0001 & 0 \\
0 & 0 & 0.0001
\end{array}\right) \quad, \quad R_{t}=\left(\begin{array}{ccc}
0.001 & 0 & 0 \\
0 & 0.001 & 0 \\
0 & 0 & 0.001
\end{array}\right),
\end{gathered}
$$

Los valores iniciales para FPSE fueron, los siguientes:

$$
\begin{gathered}
\hat{x}_{0}^{+}=(1,0,0.04) \quad, \quad P_{0}^{+}=\left(\begin{array}{ccc}
0.0001 & 0 & 0 \\
0 & 0.0001 & 0 \\
0 & 0 & 0.0001
\end{array}\right) \\
Q_{t}=\left(\begin{array}{ccc}
0.00001 & 0 & 0 \\
0 & 0.00001 & 0 \\
0 & 0 & 0.00001
\end{array}\right) \\
R_{t}=\left(\begin{array}{ccc}
0.00001 & 0 & 0 \\
0 & 0.00001 & 0 \\
0 & 0 & 0.00001
\end{array}\right) .
\end{gathered}
$$

Además para el FKSE y FPSE se tiene que: $\alpha=10^{-3}, \kappa=0$ y $\beta=2$. En la Figura (2), se muestra la morfología que describe los cinco extremos de las ondas $P, Q, R, S$, y $T$ en el círculo unitario generado por el modelo sintético dado en (43), conjuntamente con los valores estimados por los algoritmos FPG y FPR, observándose similitud entre picos simulados y los picos estimados. En la Figura 3 se muestra la misma gráfica dada en 2, pero con valores estimados usando los algoritmos FKSE, y FPSE, respectivamente. En la Figura 4 se muestra el electrocardiograma generado por el modelo sintético verdadero, conjuntamente con las medias a posteriori de los estados estimados por el FPG y FPR. En el gráfico se observa una buena aproximación de los algoritmos propuestos con respecto al modelo sintético. En la Figura 5 se muestra el modelo sintético verdadero, conjuntamente con las medias a posteriori de los estados estimados por los algoritmos FKSE y FPSE, observándose más variabilidad en los estimados con respecto a las salidas del modelo verdadero. En la Tabla 1 se muestra un resumen de la desviación estándar empírica para $N=5000$ partículas de longitud $M=200$ usando el modelo sintético del ECG y se estimó la desviación 


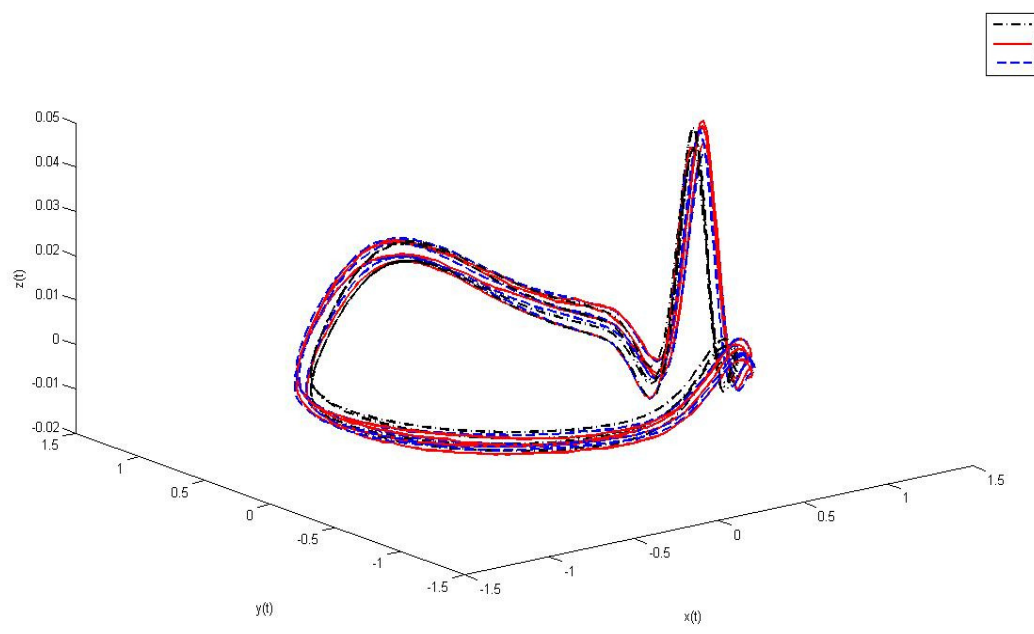

Figura 2: Reconstrucción de trayectorias: ECG sintético, FPG y FPR.

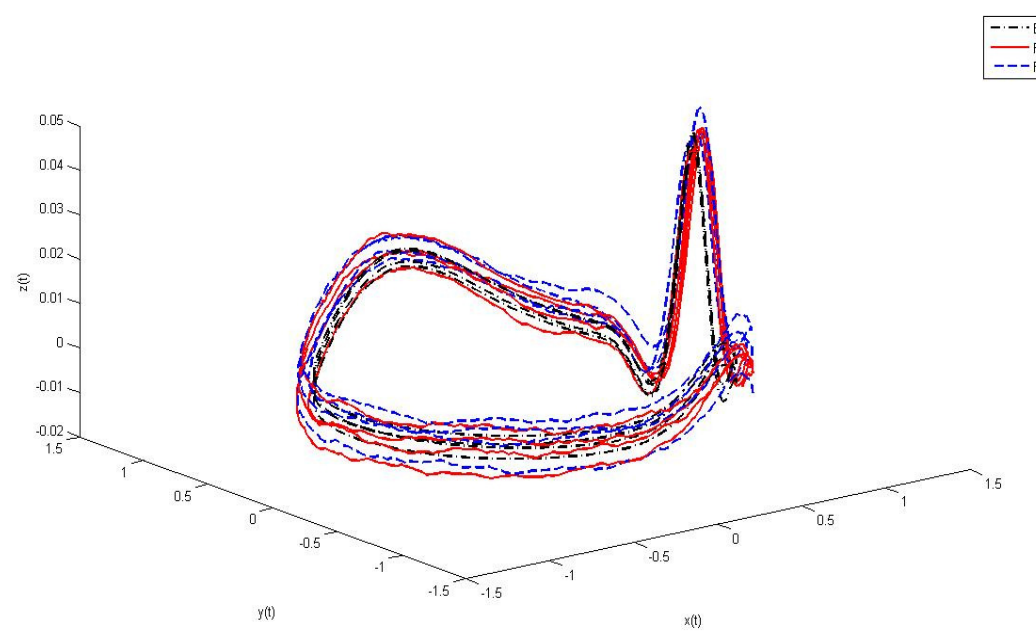

Figura 3: Reconstrucción de trayectorias: ECG sintético, FKSE y FPSE.

estándar empírica del estado de la variable de interés $z$, en la Tabla 1 no se observan diferencias significativas en los errores estimados, es decir; la diferencia 


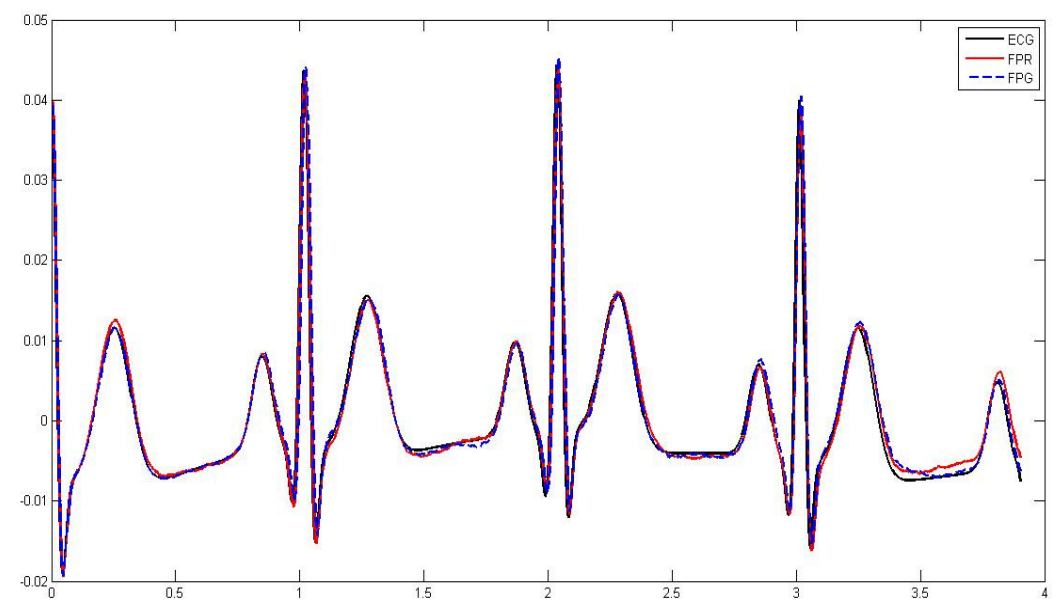

Figura 4: ECG sintético y medias a posteriori estimadas por FPG y FPR.

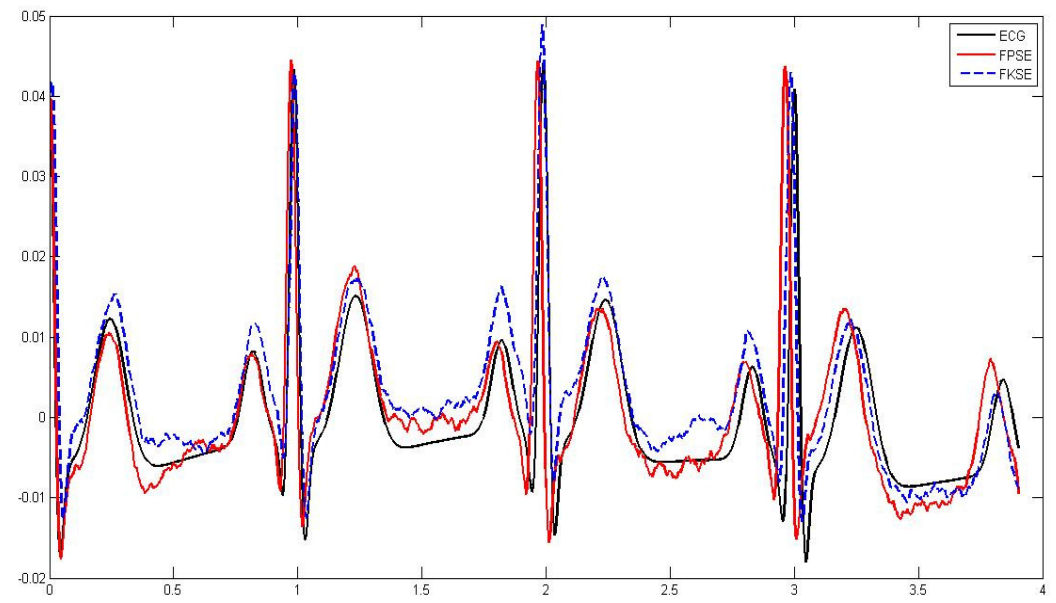

Figura 5: ECG sintético y medias a posteriori estimadas por FKSE y FPSE.

entre el valor real y el valor estimado es mínima. Posteriormente y para validar los resultados simulados, se ajustó el modelo sintético de un ECG, pero ahora 


\begin{tabular}{l|rrrr}
\hline \hline Filtros & \multicolumn{1}{|c}{ FPG } & \multicolumn{1}{c}{ FPR } & \multicolumn{1}{c}{ FKSE } & \multicolumn{1}{c}{ FPSE } \\
\hline DEE $(\mathrm{z})$ & 0.0011 & 0.0011 & 0.0075 & 0.0009 \\
CPU Time (seg) & 79.205772 & 79.934732 & 0.095665 & 547.326892 \\
\hline \hline
\end{tabular}

Tabla 1: Comparación de la desviación estándar empírica: modelo sintético simulado.

usando datos reales tomados sobre un individuo sano, donde la frecuencia de muestreo fue de $125 \mathrm{~Hz}$, la frecuencia cardíaca media fue de $1.2 \mathrm{~Hz}$ o 72 latidos por minutos. Para inicializar los FPG y FPR se consideraron los siguientes valores a priori: $x_{0}=1, y_{0}=0, z_{0}=0.04, u_{1 t} \sim N\left(0,10^{-1}\right), v_{1 t} \sim N\left(0,10^{-1}\right)$, $e_{t} \sim N\left(0,10^{-1}\right), \zeta_{t} \sim N(\mathbf{0}, 10 \mathbf{I})$. Para los algoritmos FPG y FPR se eligió una función de importancia como se elige en Arulampalam et at. (2002). Los valores iniciales a priori para FKSE fueron los siguientes:

$$
\begin{aligned}
\hat{x}_{0}^{+}=(1,0,0.04) \quad, \quad P_{0}^{+}=\left(\begin{array}{lll}
1 & 0 & 0 \\
0 & 1 & 0 \\
0 & 0 & 1
\end{array}\right) \\
Q_{t}=\left(\begin{array}{ccc}
0.1 & 0 & 0 \\
0 & 0.1 & 0 \\
0 & 0 & 0.1
\end{array}\right) \quad, \quad R_{t}=\left(\begin{array}{ccc}
0.1 & 0 & 0 \\
0 & 0.1 & 0 \\
0 & 0 & 0.1
\end{array}\right),
\end{aligned}
$$

Los valores iniciales a priori para FPSE fueron los siguientes:

$$
\begin{gathered}
\hat{x}_{0}^{+}=(1,0,0.04) \quad, \quad P_{0}^{+}=\left(\begin{array}{ccc}
0.0000001 & 0 & 0 \\
0 & 0.0000001 & 0 \\
0 & 0 & 0.0000001
\end{array}\right) \\
Q_{t}=\left(\begin{array}{ccc}
0.0000001 & 0 & 0 \\
0 & 0.0000001 & 0 \\
0 & 0 & 0.0000001
\end{array}\right), R_{t}=\left(\begin{array}{ccc}
10 & 0 & 0 \\
0 & 10 & 0 \\
0 & 0 & 10
\end{array}\right) .
\end{gathered}
$$

Además para el FKSE y FPSE $\alpha=10^{-3}, \kappa=0$ y $\beta=2$.

En la Figura 6 se muestra una representación de la señal generada por el modelo sintético con datos reales, conjuntamente con las medias a posteriori de los estados estimados por los algoritmos FPG y FPR, además en la Figura 7 se muestra la señal de ECG real, conjuntamente con las medias a posteriori de los estados estimados por los algoritmos FKSE y FPSE, en ambos gráficos se 


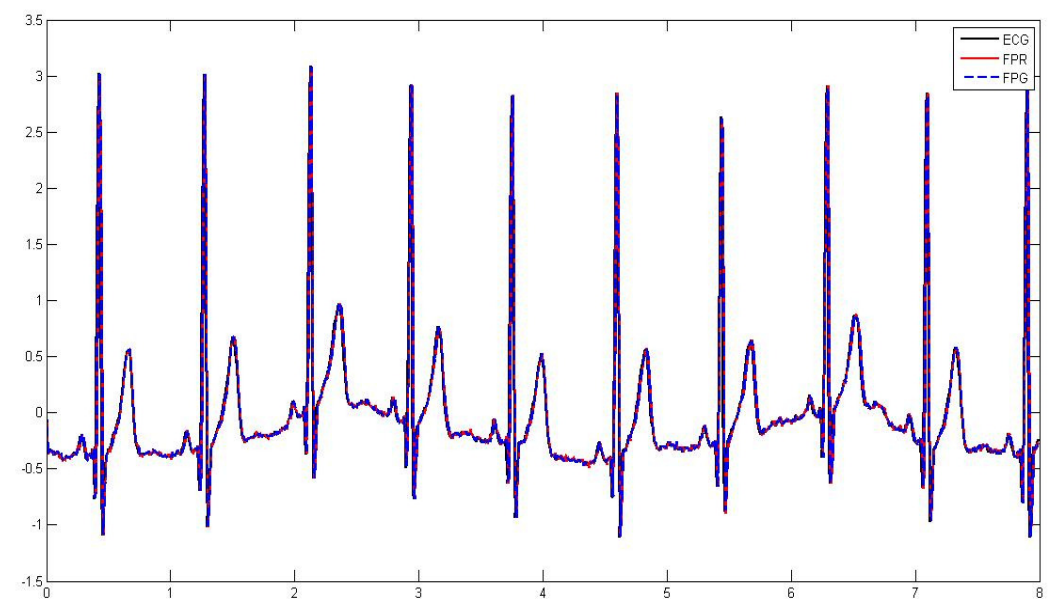

Figura 6: ECG real, FPG y FPR.

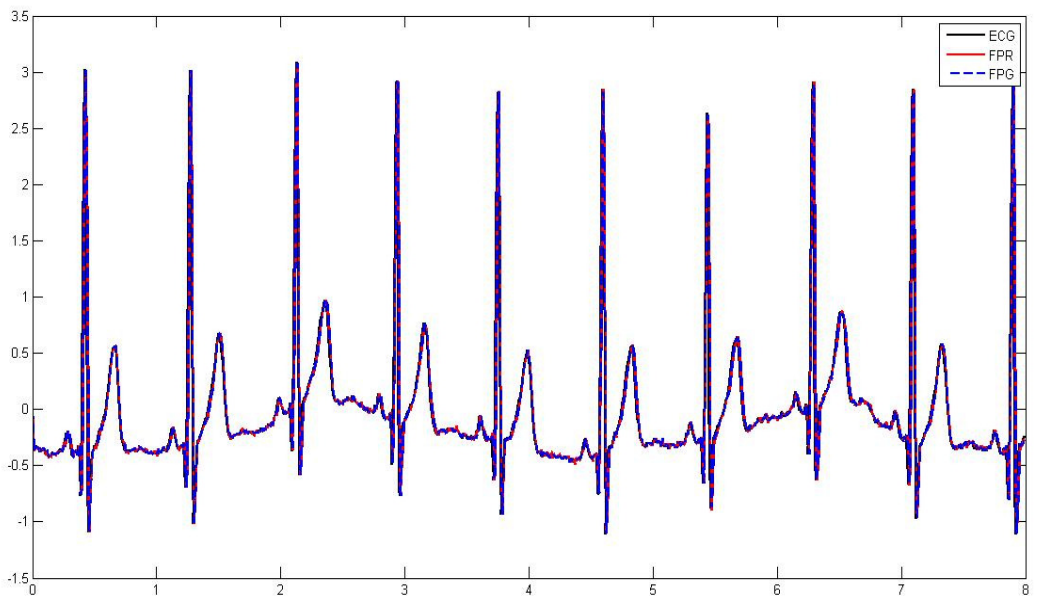

Figura 7: ECG real, FKSE y FPSE.

observa que los valores estimados y valores reales tienen el mismo patrón. En la Tabla 2 se muestra un resumen de la desviación estándar empírica para el modelo 
sintético con datos reales del ECG, no se observan diferencias significativas en los errores estimados.

\begin{tabular}{l|rrrr}
\hline \hline Filtros & \multicolumn{1}{|c}{ FPG } & \multicolumn{1}{c}{ FPR } & \multicolumn{1}{c}{ FKSE } & \multicolumn{1}{c}{ FPSE } \\
\hline DEE $(\mathrm{z})$ & 0.3359 & 0.3359 & 0.3385 & 0.3358 \\
CPU Time (seg) & 80.735960 & 80.620896 & 0.091462 & 504.150175 \\
\hline \hline
\end{tabular}

Tabla 2: Comparación de la desviación estándar empírica: modelo sintético individuo sano.

\section{Discusión y conclusiones}

La disponibilidad de modelos computacionales que simulen sintéticamente el comportamiento de las señales de los electrocardiogramas puede complementar la información experimental obtenida en la clínica sobre pacientes con problemas cardiovasculares. La utilidad de algún diagnóstico clínico basado en un electrocardiograma, recae sobre la calidad de la señal disponible. Las perturbaciones o ruidos en la señal pueden conducir a diagnósticos inadecuados y pronósticos falsos. Las técnicas de filtrado propuesta en este trabajo han sido utilizadas en otros contextos para limpiar los ruidos en las señales. En este artículo se utilizan algunas estrategias de cómputo para filtrar las señales que se generan de un electrocardiograma sintético; es decir, se implementan los algoritmos: FPG, FPR, FKSE, y FPSE, para estimar y reconstruir la morfología de las ondas generadas por el modelo sintético. También se reconstruyen las ondas generadas por señales de un electrocardiograma real tomada de un individuo sano. Los resultados demuestran que FPG, FPR, y FPSE estima los estados con exactitud, sin embargo estos filtros tienen un costo computacional mayor que el FKSE, debido al problema degenerativo de los pesos, lo cual trae como consecuencia que la varianza de los pesos de importancia tienden a incrementarse en el tiempo, pero tienen la virtud que son muy generales y no requieren de ningún supuesto sobre la distribución de las señales y la forma funcional que las genera. En contra parte, el FKSE se obtiene una estimación menos exacta, sin embargo su tiempo de cómputo es óptimo debido al menor numero de muestras requeridas por cada unidad de tiempo, además se requiere del supuesto de normalidad en la distribución de la señal. Estos algoritmos nos permiten en tiempo real cuantificar la dinámica subyacente de los estados de la señal del electrocardiograma, procesar la información de acuerdo a su generación, y a la vez permite monitorear las características morfológicas del electrocardiograma, es decir, las ondas $P, Q, R$, $S$ y $T$ del modelo sintético simulado, y del modelo sintético con datos reales. 
Finalmente se estima la desviación estándar empírica para medir el desempeño de los filtros, en todos los casos estudiados se obtuvieron valores pequeños para los errores estimados con poca variabilidad.

\section{Agradecimientos}

Esta investigación fue parcialmente financiada por el Consejo de Desarrollo Científico y Humanístico de la Universidad de Carabobo, proyecto $C D C H-$ $H M-0462-10$.

\section{Referencias}

[1] Andrieu, C; Doucet, A; Holenstein, R. (2010) "Particle Markov chain Monte Carlo methods", Journal Royal Statistical Society, B, 72(3): 269342.

[2] Arulampalam, S.; Maskell, S.; Gordon, N.; Clapp, T.A. (2002) "Tutorial on particle filters for on-line non linear/non Gaussian Bayesian tracking", IEEE Transactions on Signal Processing 50(2): 174-188.

[3] Barros, A; Mansour, A; Ohnishi, N. (1998) "Removing artifacts from electrocardiographic signals using independent components analysis", Neurocomputing 22(1-3): 173-186.

[4] Chen, Z. (2003) "Bayesian filtering: From Kalman filters to particle filters, and beyond", Technical report, Adaptive Systems Lab, McMaster University.

[5] Chui, C; Chen, G. (2009) Kalman Filtering with Real-Time Applications, Fourth Edition. Springer, Berlin.

[6] Clifford, G.; Tarassenko, L. (2001) "One pass training of optimal architecture auto associative neural network for detecting ectopic beats", Electronics Letters 37(18): 1126-1127.

[7] Doucet, A.; Godsill, S.; Andrieu, C. (2000) "On sequential Monte Carlo sampling methods for Bayesian filtering", Statistics and Computing 10: 197-208.

[8] Doucet, A.; De Freitas, J.F.G.; Gordon, N., Eds. (2001) Sequential Monte Carlo Methods in Practice. Springer Verlag, New York. 
[9] Gordon, N.; Salmond, D.; Smith, A.F.M. (1993) "Novel approach to nonlinear non Gaussian Bayesian state estimation", IEEE Proceedings $F$ (Radar and Signal Processing) 140(2): 107-113.

[10] He, T; Clifford, G; Tarassenko, L. (2006) "Application of independent component analysis in removing artefacts from the electrocardiogram", Neural Comput. Appl. 15: 105-116.

[11] Julier, S. (2000) "The scaled unscented transformation", IEEE Transactions on Automatic Control 45(3): 477-482.

[12] Julier, S; Uhlmann, J; Durrant, H. (2000) "A new method for nonlinear transformation of means and covariances in filter and estimators", IEEE Transactions on Automatic Control 45(3): 477-482.

[13] Julier S.J.; Uhlmann, J.K. (2004) "Unscented filtering and nonlinear estimation", Proceedings of the IEEE 92(3): 401-422.

[14] Kong, A; Liu, J; Wong, W. (1994) "Sequential imputations and Bayesian missing data problems", Journal of the American Statistical Association 89: $278-288$.

[15] Liu, J. (1996) "Metropolized independent sampling with comparison to rejection sampling and importance sampling", Statistics and Computing 6: 113-119.

[16] McSharry, P; Clifford, G; Tarassenko, L; Smith, L. (2003) "A dynamical model for generating synthetic electrocardiogram signals", IEEE Transactions on Biomedical Engineering 50(3): 289-294.

[17] Moody, G.; Mark, R. (1989) "QRS morphology representation and noise estimation using the Karhunen-Loeve transform", Computers in Cardiology IEEE 16th Conference, Jerusalem: 269-272.

[18] Nikolaev, N; Nikolov, Z; Gotchev, A; Egiazarian, K. (2000) "Wavelet domain Wiener filtering for ECG denoising using improved signal estimate", in: Proc. of ICASSP, IEEE Int. Conf. on Acoustics, Speech, and Sig. Proc., Vol.6: 3578-3581.

[19] Paul, J; Reddy, M; Kumar, V. (2000) "A transform domain SVD filter for suppression of muscle noise artefacts in exercise ECG", IEEE Trans. Biomed. Eng. 47(5): 654-663. 
[20] Potter M; Gadhok, N; Kinsner, W. (2002) "Separation performance of ICA on simulated EEG and ECG signals contaminated by noise", in: Canadian Conference on Electrical and Computer Engineering (IEEE CCECE), 1215 May, vol. 2: 1099-1104.

[21] Sameni, R; Shamsollahi, M; Jutten, C. (2005) "Filtering noisy ECG signals using the extended Kalman filter based on a modified dynamic ECG model", Computers in Cardiology IEEE 32nd Conference, Lyon: 10171020 .

[22] Sánchez, L., Infante, S; (2013) "Reconstruction of chaotic dynamic systems using nonlinear filters", Chilean Journal of Statistics 4(1): 35-54.

[23] Sayadi, O; Sameni, R. (2007) "ECG denoising using parameters of ECG dynamical model as the states of an extended Kalman filter", in: 29th Annual International Conference of the IEEE Engineering in Medicine and Biology Society (EMBS, Lyon 22-26 Aug. 2007): 2548-2551.

[24] Sayadi, O; Shamsollahi, M. (2008a) "ECG denoising and compression using a modified extended Kalman filter structure", IEEE Trans. on Biomedical Engineering 55(9): 2240-2248.

[25] Sayadi, O; Shamsollahi, M. (2008b) "Model-based fiducial points extraction for baseline wandered electrocardiograms", IEEE Trans. on Biomedical Engineering 55(1): 347-351.

[26] Sayadi, O; Shamsollahi, M. (2009) "A model-based Bayesian framework for ECG beat segmentation". Journal of Physiological Measurement 30(3): $335-352$.

[27] Sayadi, O; Shamsollahi, M; Clifford, G.D. (2010) "Synthetic ECG generation and Bayesian filtering using a Gaussian wave-based dynamical model”, Journal of Physiological Measurement 31(10): 1309-1329.

[28] Schreiber, T; Kaplan, D.T. (1996) "Nonlinear noise reduction for electrocardiograms", Chaos 6(1): 87-92.

[29] Simon, D. (2006) Optimal State Estimation. Kalman, $H_{\infty}$, and Nonlinear Approaches. John Wiley \& Sons, Hoboken NJ.

[30] Storvik, G. (2002) "Paticle filters in state space models with the presence of unknown static parameters", IEEE Trans. on Signal Processing 50(2): 281-289. 
[31] van der Merwe, R.; Doucet, A; de Freitas, N; Wan, E. (2000) "The unscented particle filter". Technical Report CUED/F-INFENG/TR 380, Engineering Department, Cambridge University.

[32] van der Merwe, R. (2004) Sigma-Point Kalman Filters for Probabilistic Inference in Dynamic State-Space Models. PhD. Thesis, OGI School of Science \& Engineering, Oregon Health and Science University, Portland.

[33] Vepa, R. (2010) "Nonlinear filtering of oscillatory measurements in cardiovascular applications", Mathematical Problems in Engineering 2001, Article ID 808019, 18 pages. 